\title{
Practice pitfalls
}

\author{
RUSSELl G. THORNTON, JD
}

A physician recently told me that while acting as an expert on medical-legal matters can be stressful (particularly the deposition part), expert review work was beneficial to his practice: he could see firsthand the situations that led to health care liability claims and then proactively avoid or limit those situations and, hopefully, avoid future claims. All health care providers might benefit from a brief overview of some relatively common situations seen in litigation. Here are some scenarios that we frequently see as the basis of claims.

\section{UNINFORMED PATIENT AND FAMILY}

Many lawsuits are investigated or filed because the patient or family does not understand how or why a bad outcome occurred. The problem usually boils down to the fact that the patient and/or the family, for whatever reason, did not have a good understanding of the significant nature and effect of the underlying disease and/or the risks associated with the treatment plan.

The regular success of physicians in diagnosing and treating very severe illnesses works against them when things go wrong. When there is a treatment failure or a bad outcome, the actions or inactions of the treating physician are perceived as the cause. In litigation, the patient and family may admit to having known theoretically that a bad outcome could occur but never seem to admit that they appreciated that it could occur in their particular situation.

For this reason it is important for the health care team to document that the patient was advised about the severe nature of his or her disease and its associated morbidity and mortality. Further, the documentation should note that the patient was advised of the treatment options (it is a good idea to list the options discussed), that the patient understood the risks associated with those options (list the significant risks), and that the patient elected to proceed. If family members were present at the discussion, that should be noted in the chart as well.

This type of documentation is also important in defending a claim that the treatment provided was not indicated based on the patient's clinical condition. Notation of the specific indications for intervention gives the jury something more to consider than the defendant's testimony when resolving this particular criticism.

While we understand that physicians have legitimate concerns about the need to document "everything," documentation is not necessarily time intensive. For example, a note can state something to the effect of "Mr. Smith was advised that he suffers from $\mathrm{X}$ and understands that, untreated, this condition will likely result in $\mathrm{A}$ and $\mathrm{B}$. Mr. Smith was informed that treatment options 1,2 , and 3 are potential interventions to cure/treat $\mathrm{X}$ and/or address $\mathrm{A}$ and/or B. Treatment options 1, 2, and 3 were discussed, including their risks and benefits. After considering each option, Mr. Smith elected to proceed with 3, understanding the risks of $\mathrm{X}, \mathrm{Y}$, and $\mathrm{Z}$ associated with this intervention." This "note," which took less than 2 minutes to write, is a valuable piece of evidence that can be put before every witness in deposition or at trial to support the contention that the patient was well informed and aware of all pertinent facets of the situation.

\section{PREOPERATIVE CLEARANCE}

An allegation that the patient should not have been cleared for some form of "elective" or nonemergent surgery is also common when the surgical outcome is poor. In these situations, the patient frequently has a significant preexisting cardiac or pulmonary problem that is the cause of the outcome. Everyone involved in the patient's preoperative evaluation and care is generally named as a defendant in these claims on the basis that surgery should not have gone forward. Regardless of the actual medical nature of the complication, the anesthesiologist, the primary care physician, and any consultants, such as a cardiologist or pulmonologist, are defendants. The defense is frequently left to address a claim that the physician provided some type of "global" surgical clearance, even though he or she addressed only one facet of the patient's underlying condition.

Physicians involved in the preoperative clearance of a patient need to document their specific role and actions in the evaluation of the patient and their specific evaluation/opinion/recommendation. For example, the primary care physician who wants a cardiac and/or pulmonary consultation because of an underlying problem needs to document this fact. If he or she clears the patient for surgery because of the opinion/consultation of a specialist, this fact needs to be documented. Likewise, a consulting specialist needs to document that he or she is addressing only a single aspect of the patient's condition.

The absence of this type of documentation places a defendant somewhat at the mercy of his fellow physicians to accurately

From Stinnett, Thiebaud \& Remington, LLP, Dallas, Texas.

Corresponding author: Russell G. Thornton, JD, Stinnett, Thiebaud \& Remington, LLP, 4800 Fountain Place, 1445 Ross Avenue, Dallas, Texas 75202 (e-mail: rthornton@strlaw.net). 
describe and establish his role in the patient's care. If fellow physicians "run for cover" and make a defendant's role in the case bigger than it really was, the defendant has nothing other than his word to controvert this testimony and back up his position that his role was limited.

\section{NEWER THERAPEUTIC OPTIONS}

With new medical interventions and surgical techniques coming at a relatively fast pace, a decision to use something beyond a "tried-and-true" treatment needs to be clearly documented. If not, rest assured, if there is a bad outcome, a complication from the treatment, or a treatment failure, the patient will claim that he was not aware a new intervention was being used and that if he knew this fact he would have selected the "tried-and-true" intervention.

The trade-off for newer therapies is generally a lower morbidity risk (either intuitively or established) for a potential lower chance of success or remedy. Oftentimes, the decision is not so much medical as one of patient preference (Do you want to trade less postoperative pain for a slightly lower chance of success?). Examples of these situations include laparoscopic procedures (though less so now) and other minimally invasive interventions, such as off-pump coronary artery bypass surgery and minimally invasive coronary artery bypass surgery and thoracic surgery.

In these situations, the record should clearly show that the patient was advised of the specific options of $A$ and $B$; knew that $B$ is newer, has potentially less morbidity (describe the morbidity at issue), but has a potentially lower chance of success; and after considering these matters elected to proceed with option B. Further, the consent form/operative permit should clearly indicate the specific procedure to be performed. For example, if an off-pump bypass surgery is being performed, the best practice is to state this on the consent form, as opposed to a simple statement that the surgery is coronary artery bypass graft surgery.

\section{TEST RESULTS}

The failure to communicate and be aware of important test results is very problematic. For example, a pathology report or radiology study may show that a patient has cancer, and this information is not quickly or effectively communicated to and/or appreciated by the patient's treating health care providers. Because the clinician does not know this information, timely procedures to diagnose and/or treat the problem are not performed. The patient may die as a result.

In our experience, what happens is that instead of calling or otherwise directly communicating with the clinician, the radiologist or pathologist simply mentions a suspicion of a significant disease in his or her report and puts the report in the patient's hospital chart or mail in the normal course of business. The report then either does not make it to the hospital chart before discharge, is never received by the clinician's office, or is not brought to the clinician's attention after it is received by his office. This situation creates significant liability exposure for all involved health care providers. The radiologist/pathologist is sued for failing to properly communicate the results to the clinician, and the clinician is sued for failure to know important test results.

Further complicating matters, the radiologist/pathologist and the clinician (and sometimes the clinician and his office person- nel) get involved in finger-pointing. The pathologist/radiologist says that the results were available for review by the clinician, and the clinician says that he expected some form of direct contact from the pathologist/radiologist for this type of significant finding. To a certain extent, both may be right. However, finger-pointing does nothing other than create exposure for all involved, increase the value of the patient's claim (since juries do not seem to like defenses that claim that someone else was wrong), and make it easier for the plaintiff to prevail and obtain a significant verdict. In these situations, all the patient has to do is tell the jury that someone clearly made a mistake (since each doctor blames the other) and that they only need to determine who erred and to determine the value of the error. In fact, since the doctors blame each other and admit that the failure to quickly intervene damaged the patient, the patient often does not even need an expert witness to establish liability and causation. The defendants provide that evidence themselves.

To avoid this problematic situation, the pathologist/radiologist should directly contact the clinician with significant test results either through a personal phone call or a fax of the report. If done, this action needs to be documented.

Clinicians need a tickler system to ensure review of test results. One method is to schedule an appointment to review test results and their implications with the patient. When there is an office visit for that purpose, there is obviously a review of the chart to see the test results. If the results are not in the chart, for whatever reason, they can be quickly obtained, and nothing is overlooked. Second, there must be a procedure for the physician to review all abnormal test results and for a health care provider to initial test results before they are placed in the patient's chart. Then no results are filed in the chart without some health care provider in the office knowing what they are and a nonphysician will not misinterpret the significance of a particular patient's abnormal results.

\section{PATIENT TRANSFERS}

Circumstances surrounding the transfer of patients from one facility to another are also a frequent issue in health care liability claims. In these cases, the patient was usually transferred because the first facility (often outside a major metropolitan area) did not have the staff or equipment to properly evaluate and/or treat the patient's condition.

These claims often focus on the attending physician's inattention to the transfer once it has been requested. Frequently, although the transferring physician promptly recognizes the need for transfer, the actual transfer is delayed, which results in significant morbidity or mortality. Generally, the delay is caused when a physician selected by the transferring doctor will not or cannot immediately accept the patient or when the facility where the receiving physician has staff privileges does not have room for the patient at the time of the transfer.

While a physician may properly believe that the mechanics of the transfer are handled hospital-to-hospital, the fact remains that the attending physician at the transferring facility remains responsible for the patient until the patient is received at the new facility. Given this situation, the transferring physician needs to keep two things in mind. First, how urgent is the need for the patient to be at a different facility? Second, can another physician 
or facility properly evaluate and treat the patient? If the need is urgent, the physician should consider either 1 ) seeing if a different physician at a different facility will accept the patient or 2) advising the receiving facility that the transfer is emergent. In emergent situations, the receiving facility may be able to "make space" for the patient. If the need is not urgent and the patient is stable, it is reasonable and proper to wait for the desired facility to "open up." These facts and thought processes, however, should be documented in the record. A failure to consider these matters, act accordingly, and document this information opens the transferring physician to a claim that alternative options would have allowed the patient to receive the needed care in a more timely fashion and avoid a poor outcome.

\section{DEALING WITH BAD OUTCOMES}

Health care liability claims are more likely when the patient, or the patient's family, feels that the physician was evasive or cursory in responding to their inquiries about a bad outcome. While bad outcomes are never pleasant to discuss, the physician must deal with the patient and/or the family in a candid, timely, and proper manner. In our experience, a good practice is to schedule an appointment with the patient/family to discuss the situation after the "crisis" has passed. This is by no means a foolproof manner of avoiding a lawsuit, but it certainly provides the physician an opportunity to defuse a confusing situation and allows defense counsel to argue that the physician was being forthright and candid about the situation and was not trying to hide anything.

\section{FAILURE TO FOLLOW INSTINCT}

Health care providers should trust their instincts. In countless initial meetings, clients have informed counsel that they knew that the patient had unrealistic expectations or knew the patient would be unhappy regardless of their efforts. If physicians really believe that the patient will not be happy with the results, they should not go forward with an elective procedure. If the treatment at issue is medically necessary, they should clearly document the patient's unrealistic expectations in the record.

\section{THEMES TO REMEMBER}

Some common themes run through most practice pitfalls. First, documentation is important. Health care providers should now be well aware that attorneys and their peers frequently review their conduct. Everyone recognizes the significant risk of litigation that stems from any bad outcome. Further, as we pointed out in a recent article in this publication, licensing agencies such as the Texas State Board of Medical Examiners have become more aggressive in their investigation and review of health care providers (1). Given these realities, the best evidence for a physician's defense is the documentation from interactions with and treatment of the patient. This information is usually the first thing reviewed. As such, it provides the reviewer with a first and lasting impression of the physician and the patient. Good and thorough documentation creates an initial good impression, while poor, incomplete documentation creates a poor impression. These initial impressions may go a long way in determining whether or not a claim or inquiry is pursued. Thus, the importance, effectiveness, and utility of good documentation cannot be overestimated.

Second, timely, effective, and accurate communication with the patient, and between the patient's health care providers, is key. Complete, open, and candid communication with the patient can eliminate one reason claims are frequently filed. Thorough communications between a patient's health care providers may not only help to avoid claims but also clarify a physician's role in the patient's care and prevent him from being "roped in" by the patient and/or colleagues in areas of the patient's care for which he was not responsible. These efforts can often be the difference between avoiding litigation and defending a problematic liability claim.

Third, a physician's relationship with the patient is often an underappreciated element of the "art" of practicing medicine. An honest and strong relationship with the patient, and the patient's family when indicated and appropriate, can go a long way in avoiding litigation and resolving litigation on favorable terms when it does occur. When a strong relationship exists, our experience is that the patient, or the patient's family, has a better perception of the physician and his or her efforts to treat the patient. The existence of a poor relationship with the patient not only increases the likelihood of litigation but results in an even greater schism between the physician and the patient if litigation does occur, which thereby creates another obstacle to the favorable resolution of a claim.

There is one last thing to point out about effective documentation. It is not unusual for even the best health care provider to come across poorly in deposition or trial for whatever reason. When that happens, opposing counsel and the jury may question the credibility of the testimony and the position of the health care provider. Documentation can remove many of the problems that can result from poor presentation. With good documentation, the defendant's position is contained in a document created before litigation and can be shown to each witness at trial. It provides additional evidence (and really the primary evidence) to support the defendant's position and is something that the jury can review and take back to the jury room with them in determining the defendant's fate.

1. Thornton RG. Handling investigations of the Texas State Board of Medical Examiners. BUMC Proceedings 2004;17:374-376. 